\title{
Research Paper: Adverse Drug Reaction Monitoring of Anticancer Drugs in Hematology Department
}

\author{
Ghazal Roohi ${ }^{1}$, Panner Selvam R ${ }^{1}$, Fares Najari ${ }^{2 *}$
}

1. Department of Pharmacy Practice, T.John College of Pharmacy, Rajiv Gandhi University of Health Sciences, Karnataka ,Bengaluru, India.

2. Department of Legal Medicine, Faculty of Medicine, Shahid Beheshti University of Medical Sciences, Tehran, Iran

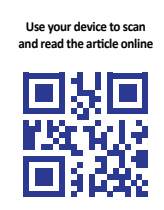

Citation: Selvam P, Roohi G, Najari F. Adverse Drug Reaction Monitoring of Anticancer Drugs in Hematology Department. International Journal of Medical Toxicology and Forensic Medicine. 2021; 11(3):32776. https://doi.org/10.32598/ijmtfm.v11i3.32776

https://doi.org/10.32598/ijmtfm.v11i3.32776

Article info:

Received: 4 Nov 2020

First Revision: 14 Dec 2020

Accepted: 18 Feb 2021

Published: 3 Oct 2021

\section{Keywords:}

Adverse drug reaction, Anticancer agents, Active surveillance, Acute Leukemic Leukemia

\section{A B S T RACT}

Background: Cancer is among the leading causes of mortality in India. Studies have reported antineoplastic agents as the common class of drugs causing Adverse Drug Reactions (ADRs). The present study aimed to conduct active surveillance of ADRs of anticancer drugs in the hematology department.

Methods: A prospective observational study was conducted in 136 patients with cancer and the incidence and frequency of ADRs were assessed. The study was conducted in 6 months in a multispecialty hospital.

Results: Among 136 cancer patients, All was more prevalent (39.70\%); CLL, Non- Hodgkin's Lymphoma were less prevalent $(0.73 \%)$. ADRs were more prevalent in the Pediatrics department, i.e., $18.53 \%$ of ADRs were observed in patients aged $<10$ years. ADRs in male patients constituted $54.39 \%$, whereas it was $45.60 \%$ in female patients. Cytarabine caused the highest number of ADRs (34.48\%). The most prevalent ADR was anemia (25.60\%).

Conclusion: Multiple ADRs were detected in cancer patients. We found that hematological ADRs were more prevalent. Most of the ADRs were possible reactions according to Naranjo and the World Health Organization (WHO) scales

\section{T}

\section{Introduction}

he development and use of drugs in various diseases have brought wonders to the human race; however, it has also set new threats in the form of Adverse Drug Reactions (ADRs) [1]. An ADR is defined as any response to a drug, i.e., noxious, unintended, and occurs at doses used in man for prophy- laxis, diagnosis, or therapy. [2-4]. Worldwide, ADRs accounted for $10 \%$ of hospital admissions [5] and $6 \%$ of hospitalized patients encountered ADRs [6, 7]. The most commonly used method for post-marketing surveillance is ADR monitoring. The methods of ADR monitoring are as follows:

1. Spontaneous reporting of ADR

2. Intensive hospital-based cohort studies

"Corresponding Author:

Fares Najari, MD.

Address: Department of Legal Medicine, Faculty of Medicine, Shahid Beheshti University of Medical Sciences, Tehran, Iran.

Tel: +98 (912) 3195140

E-mail: najari.hospital@sbmu.ac.ir 


\section{Case-Control surveillance}

\section{Prescription event monitoring}

The Pharmacovigilance Program of India (PVPI) is in collaboration with the WHO-UMC. Sweden is working to contribute the Indian data to the international database. The aims of PVPI are as follows:

- To monitor ADRs in the Indian population

- To create awareness among healthcare professionals about the importance of ADRs

reporting in India

- To generate independent evidence-based recommendations on the safety of medicines

- To monitor benefit-risk profiles of medicines among others [8-10].

With the causality assessment of ADRs, we can estimate the strength of the relationship between drug exposures and the occurrence of adverse reactions. In this study, Naranjo and the WHO scales were used for causality assessment. The most common ADRs detected in cancer patients receiving anticancer agents include the following: alopecia, nausea, vomiting, burning sensation, tingling, fatigue, anorexia, anemia, thrombocytopenia, leucopenia, ulceration, fever, and bone marrow suppression [7, 11, 12]. The common anti-cancer drug which manifests ADRs are rituximab, imatinib, bortezomib, thalidomide, methotrexate, 5-fluorouracil, cisplatin, paclitaxel, adriamycin, leucovorin, oxaliplatin, and etoposide [13-15].

\section{Materials and Methods}

This was a prospective and applied study in terms of data collection. In total, 136 cases with hematological cancers were selected from the oncology department in a tertiary care hospital in Bangalore City, India, for 6 months. The study subjects were selected using a simple random sampling method and according to the inclusion criteria of the study. Then, to ensure the significance of the findings, we considered the relevant criteria after calculating the minimum sample size using the Cohen formula. The study patients provided informed consent forms.

The inclusion criterion of the study included all types of hematological cancer patients of both genders. The excluded criteria of the study were as follows: Cases without hematological cancers; cases without a previous disease history, and pregnant women.

The following ethical principles were also observed in this research: Physicians' and patients' names remained confidential and the demographic details of the study patients remained confidential.

The standard organization form, containing the demographic details of the patients, social habits, medical history, medication history, current medication with any ADRs and interactions, vitals, and signs was used in this research.

Descriptive statistics were used to describe the research results. The frequency and percentage of qualitative variables, as well as Confidence Intervals (95\% $\mathrm{CI}$ ), mean, and standard deviation values were reported, subsequently. Chi-squared test and Student's Samples t-test were used to analyze the research findings in SPSS. $\mathrm{P}<0.05$ was considered as the significance level. Moreover, The Ethics Committee approved this study (Code: 258/A, on 24 ${ }^{\text {th }}$ Jan. 2019 at Narayana Hrudayalaya Ltd). The listed drugs and malignancies types were as follows:

ALL: MMF, Cytarabine, Leflunomide, Cyclophosphamide, Doxorubicin, L-Asparaginase, 6-MP, Methotrexate, Vincristine, Daunorubicin, Prednisolone, Teicoplanin, Vinblastin

AML: Daunorubicin, Cytarabine, Decitabine Idarubicin, Tacrolimus, Dexamethasone, Prednisolone, Valganciclovir, Doxorubicin

APML: Daunorubicin, Cytarabine, Fludarabine

CLL: Rituximab, Fludarabine, Cyclophosphamide

Hodgkin Lymphoma: Cytarabine, Vinblastine, Bleomycin, Doxorubicin

Non-Hodgkin Lymphoma: Etoposide, Rituximab

Lymphoma: Rituximab, Cyclophosphamide, Vincristine, Doxorubicin, Temozolamide, Methotrexate, Cytarabine

Multiple Myeloma: Bortezomib, Daratumumab, Melphalan, Dexamethasone, Thalidomide

Acute Leukaemia: Azathioprine, Cyclophosphamide, Methotrexate, L- Asparaginase 
CML: Tigecycline, Daunorubicin, Cytarabine, Methotrexate, Nilotinib, Fludarabine, Vinblastine

\section{Results}

Of the explored 136 cancer patients who were receiving chemotherapy, 92(67\%) were male and 44(32.3\%) female. The Mean \pm SD age of the study subjects was $40 \pm 30$ years. A total of 410 ADRs were recorded and analyzed. For example, acute lymphoblastic leukemia was more prevalent in children $(39.70 \%)$; chronic lymphoblastic leukemia and non-Hodgkin lymphoma were less prevalent $(0.7 \%)$. ADRs were more common in pediatrics patients $(18.53 \%)$. ADRs in male patients constituted $54.39 \%$, whereas this rate equaled $45.60 \%$. in female patients. Cytarabine caused the highest number of ADRs (34.48\%). The highest frequency of ADRs was contributed with blood disorders $(49.5 \%)$ and the lowest number of that concerned urinary system disorders $(0.7 \%)$, neurological disorders $(0.7 \%)$, and vision disorders $(0.7 \%)$.

According to Table 1, the most common cancer for those aged $<10$ years, was acute lymphoblastic leukemia (40\%). It was more prevalent in females $(61.11 \%)$ than males $(38.18 \%)$. The highest number of ADRs was due to cytarabine $(31.11 \%)$ which caused anemia $(16.35 \%)(\mathrm{P}<0.05)$.

According to Table 1, acute leukemia was more prevalent in patients aged $<10$ years $(60 \%)$. It was more frequent in female patients $(80 \%)$ than male patients
(20\%). The maximum number of ADRs concerned methotrexate and cyclophosphamide and the most prevalent ADR was leucopenia $(21.43 \%)(\mathrm{P}<0.05)$.

According to the graffiti number, acute myeloblastic leukemia was further detected in age group of 41-50 years $(25 \%)$; it was more prevalent in females $(56.2 \%)$ than males $(46.8 \%)$. The highest number of ADRs was due to cytarabine $(55.28 \%)$ and the most common ADR was anemia $(17.74 \%)(\mathrm{P}<0.05)$.

According to the graph numbers, APML and CALL were more common in females $(66.66 \%)$. In APML, fludarabine $(50.01 \%)$ presented the highest ADR with the greatest number of ADRs, such as anemia, leucopenia, and acute kidney injury; however, in chronic lymphoblastic leukemia, cyclophosphamide (54.01\%) manifested the maximum ADRs with the highest incidence of anemia, thrombocytopenia, cough, fever, nausea, and vomiting $(\mathrm{P}<0.05)$.

According to the obtained data, chronic myeloblastic leukemia was more prevalent in patients aged $41-50$ years $(50 \%)$; it was more prevalent in male patients $(75 \%)$ than females $(25 \%)$. Cytarabine indicated the highest ADRs (47.61\%) with anemia (23.81\%) $(\mathrm{P}<0.05)$.

Moreover, Hodgkin lymphoma was more common in those aged $<10$ years $(50 \%)$; it was more prevalent in males $(66.66 \%)$ than females $(33.334 \%)$. The highest ADR was due to cytarabine $(57.14 \%)$, with the domi-

Table 1. The incidence and frequency of Adverse Drug Reactions (ADRs) (N=136)

\begin{tabular}{cc}
\hline Cancer Type & No.(\%) \\
\hline Acute leukaemia & $5(4)$ \\
\hline ALL & $54(40)$ \\
AML & $32(24)$ \\
APML & $6(4)$ \\
\hline CLL & $1(1)$ \\
\hline CML & $8(6)$ \\
\hline Hodgkin's lymphoma & $6(4)$ \\
\hline Lymphoma & $11(8)$ \\
\hline Multiple myeloma & $12(9)$ \\
\hline Non-Hodgkin's lymphoma & $1(1)$ \\
\hline
\end{tabular}


Table 2. The age-wise Adverse Drug Reactions (ADRs) distribution in the study subjects ( $N=136)$

\begin{tabular}{cccc}
\hline SI. No. & Age Group (y) & No. of Patients & No. of ADR \\
\hline 1 & $<10$ & 33 & 108 \\
\hline 2 & $11-20$ & 19 & 58 \\
\hline 3 & $21-30$ & 13 & 47 \\
\hline 4 & $31-40$ & 13 & 63 \\
\hline 5 & $41-50$ & 23 & 53 \\
\hline 7 & $51-60$ & 24 & 32 \\
\hline
\end{tabular}

nation of nausea and vomiting $(23.57 \%)$ as adverse effects $(\mathrm{P}<0.05)$.

According to Table 1, lymphoma was more prevalent in patients aged $>60$ years $(36.36 \%)$ and more prevalent in males $(81.81 \%)$ than females $(18.18 \%)$. Methotrexate presented maximum ADRs $(28.57 \%)$ with leucopenia $(14.29 \%)(\mathrm{P}<0.05)$.
According to Graph 2, multiple myeloma was more prevalent in patients aged 51-60 years (75\%) and more common in male patients $(58.33 \%)$ than females $(41.66 \%)$. The highest number of ADR belonged to bortezomib (32\%) and neutropenia $(16 \%)(\mathrm{P}<0.05)$.

Table 1 presents that non-Hodgkin lymphoma was observed in only one patient from the 21-30-year-olds

Table 3. The organ system-wise distribution of Adverse Drug Reactions (ADRs) (N=136)

\begin{tabular}{ccc}
\hline SL. NO. & Organ System & No. of ADRs \\
\hline 1 & Blood disorders & 203 \\
\hline 2 & General disorders & 37 \\
\hline 3 & Gastrointestinal disorders & 23 \\
\hline 4 & Skin diseases & 22 \\
\hline 5 & Immune disorders and infections & 17 \\
\hline 6 & Liver And Biliary Disorders & 12 \\
\hline 7 & Bleeding disorders & 9 \\
\hline 8 & Respiratory system disorders & 8 \\
\hline 10 & CVS disorders & 8 \\
11 & Metabolic and nutritional disorders & 5 \\
12 & CNS and PNS disorders & 3 \\
\hline 15 & Mucoskeletal disorders & 3 \\
\hline
\end{tabular}


Table 4. The drug-wise Adverse Drug Reactions (ADRs) distribution in the study subjects ( $N=136)$

\begin{tabular}{|c|c|c|}
\hline SL.No. & Drug & No. of ADR \\
\hline 1 & 6-MP & 5 \\
\hline 2 & Azathioprine & 2 \\
\hline 3 & Bleomycin & 2 \\
\hline 4 & Bortezomib & 8 \\
\hline 5 & Cyclophosphamide & 20 \\
\hline 6 & Cytarabine & 140 \\
\hline 7 & Daratumumab & 4 \\
\hline 8 & Daunorubicin & 30 \\
\hline 9 & Decitabine & 21 \\
\hline 10 & Dexamethasone & 13 \\
\hline 11 & Doxorubicin & 15 \\
\hline 14 & Etoposide & 1 \\
\hline 12 & Fludarabine & 5 \\
\hline 13 & Idarubicin & 1 \\
\hline 15 & L-Asparginase & 3 \\
\hline 16 & Leflunamide & 6 \\
\hline 17 & Melphalan & 5 \\
\hline 18 & Methotrexate & 60 \\
\hline 19 & MMF & 9 \\
\hline 20 & Nilotinib & 2 \\
\hline 21 & Prednisolone & 2 \\
\hline 22 & Rituximab & 8 \\
\hline 23 & Tacrolimus & 4 \\
\hline 24 & Thalidomide & 4 \\
\hline 25 & Tigecycline & 1 \\
\hline 26 & Timozolomide & 4 \\
\hline 27 & Valganciclovir & 1 \\
\hline 28 & Vinblastine & 5 \\
\hline 29 & Vincristine & 25 \\
\hline
\end{tabular}


age group and was male. The highest ADRs concerned rituximab (80\%); malaise, myalgia, abdominal pain, increased LET and LDH $(100 \%)(\mathrm{P}<0.05)$.

According to Table 2, the most common cancer for those aged $<10$ years, was acute lymphoblastic leukemia $(40 \%)$. It was more prevalent in females $(61.11 \%)$ than males $(38.18 \%)$. The highest number of ADRs was due to cytarabine $(31.11 \%)$ which caused anemia $(16.35 \%)(\mathrm{P}<0.05)$.

\section{Discussion}

The identification and treatment of ADRs are among the main aims for preventing further complications in cancer patients. After ADRs were collected and analyzed, we observed that the population belonging to the Mean \pm SD age group of $40 \pm 30$ years were more prone to developing ADRs during cancer.

Chemotherapy can be compared with one study conducted in Bangladesh [13]. The incidence of ADRs was greater in male patients in ALL cancer $(45.50 \%)$, compared with female patients in AML cancer (43.85\%); it can be explained by the higher incidence of blood cancer $(22.86 \%)$ in our study, i.e., in line with those reported by the Western and Eastern part of India [1]. However, studies from other parts of India and Bangladesh identified a higher preponderance of ADRs in cases affecting female patients [15]. Cytarabine, Methotrexate, cyclosporine, and fludarabine-containing regimens were commonly used and associated with most of the ADRs in our setting $[15,16]$. The most frequently affected system by ADRs was the blood system. A $25.2 \%$ prevalence rate of anemia and thrombocytopenia was reported in our setting, i.e., significantly lower, compared with $31.5 \%$ and $48.1 \%$ stated in other studies [15]. Moreover, a 9\% nausea and vomiting was reported, i.e., significantly lower, compared with $31.5 \%$ and $48.1 \%$ were reported in two other studies [7, 17]. The lower incidence of these adverse reactions is accounted for the anti-emetic drugs, like granisetron and the administration of pantoprazole, dexamethasone, and aprepitant (Tables 1 and 2). Evidence suggested the occurrence of constipation with an anticancer regimen containing cisplatin,

5- FU, and cyclophosphamide. Constipation was very low $(0 \%)$ in our patients, compared with other studies $[4,15]$. They managed this problem with laxatives and proper counsel on dietary modification. We observed that the drug regimen comprising cytarabine, doxorubicin, vincristine, and cyclophosphamide administered especially for the treatment of lymphoma and acute lymphoblastic leukemia was associated with hematological disorders (49\%), namely anemia, neutropenia, and leucopenia [14]. Another prospective study among the patients with non-Hodgkin lymphoma treated with the same regimen reported life-threatening neutropenia $[18,19]$. In contrast to other evaluations, our study suggested hematological disorders as the most common ADRs.

In our study, all patients encountered alopecia, a figure which is less, compared with $51 \%$ and $58 \%$, i.e., reported in previous studies [20]. On analyzing the causality assessment of the ADRs by the Naranjo scale, we found that $44 \%$ of cases presented probable, and $55 \%$ indicated possible associations, whereas the WHO scale reported a $35 \%$ of the probable score. This finding was comparable with the studies conducted in southern India. Assessing the preventability scales indicated that only (44\%) of ADRs could have probably scores in patients who manifested the symptoms of anemia, nausea, vomiting, fever, and constipation; appropriate medication was given and proper dietary counsel was ensured before the onset of chemotherapy. All reported ADR cases in our study were managed symptomatically but not all recovered fully.

\section{Conclusion}

Pharmacovigilance is an essential assessment for approving safety and efficacy in medications. Analyzing ADRs associated with the anticancer drugs in a tertiary hospital provides information regarding the causality, severity, and preventability of the identified ADRs. It can provide awareness for a physician to prevent further occurrence of similar ADRs in the same patients. (Table 3).

\section{Ethical Considerations}

\section{Compliance with ethical guidelines}

All information obtained from patients Will remain confidential. the study conducted under Narayana Health Academic Ethics Committee(Code: ECR/772/ Inst/KA/2016) valid till date 28 February 2019 issued under Rule 122DD of the Indian Drugs and Cosmetics Roles 1945. 


\section{Funding}

This research did not receive any grant from funding agencies in the public, commercial, or non-profit sectors.

\section{Author's contributions}

All authors equally contributed to preparing this article.

\section{Conflict of interest}

The authors declared no conflict of interest.

\section{Acknowledgements}

We would like to express our special thanks to Dr. Ganesh. N.S. (M.pharm, PhD, Professor and HOD, T. John College of Pharmacy), Dr.Vineeth Chandy (M.pharm, PhD principal of The. John College of Pharmacy) for their valuable assistance and helpful comments throughout the research work.

\section{References}

[1] Goyal YN, Solanki KC, Mistry RA, Joshi ND, Singh AP, Gajera MV. Pattern of adverse drug reactions due to cancer chemotherapy in tertiary care teaching hospital in Gujarat. Int J Sci Res. 2014; 3(1):333-5. [DOI:10.15373/22778179/ JAN2014/112]

[2] Singh S, Dhasmana D, Bisht M, Singh PK. Pattern of adverse drug reactions to anticancer drugs: A quantitative and qualitative analysis. Indian J Med Paediatr Oncol. 2017; 38(2):140-5. [DOI:10.4103/ijmpo.ijmpo_43_16] [PMID] [PMCID]

[3] WHO. The importance of pharmacovigilance safety monitoring of medicinal products. Geneva: World Health Organization; 2002. https://apps.who.int/iris/bitstream/ handle/10665/42493/a75646.pdf

[4] Routledge PA, O'mahony MS, Woodhouse KW. Adverse drug reactions in elderly patients. Br J Clin Pharmacol. 2004; 57(2):121-6. [DOI:10.1046/j.1365-2125.2003.01875.x] [PMID] [PMCID]

[5] Farcas A, Bojita M. Adverse drug reactions in clinical practice: A causality assessment of a case of drug-induced pancreatitis. J Gastrointestin Liver Dis. 2009; 18(3):353-8. [PMID]

[6] Raut A, Patel P, Patel C, Pawar A. Preventability, predictability and seriousness of adverse drug reactions amongst medicine inpatients in a teaching hospital: A prospective observational study. Int J Pharm Chem Sci. 2012; 1(3):12939. https://ijpcsonline.com/archives3/
[7] Seenivasan P, Anandan S, Vivek PI, Rajanandh MG Adikrishnan S. Pattern of adverse drug reactions in a regional pharmacovigilance center of a tertiary care teaching hospital. Drugs Ther Perspect. 2018; 34(3):138-41 [DOI:10.1007/s40267-017-0474-y]

[8] Chopra D, Rehan HS, Sharma V, Mishra R. Chemotherapyinduced adverse drug reactions in oncology patients: a prospective observational survey. Indian J Med Paediatr Oncol. 2016; 37(1):42-6. [DOI:10.4103/0971-5851.177015] [PMID] [PMCID]

[9] Kishore P, Meghana G, Reddy BP, Raghavaiah K, Kumar DS. Pattern of adverse drug reactions and its management in female cancer patients in a private hospital in Telangana, India. Asian J Pharm Res Dev. 2018; 6(4):45-53. [DOI:10.22270/ajprd.v6i4.381]

[10] Sunny S, Thampi A, Johnkennedy NS, Babasahib SK, Chacko C. Assessment of adverse effects of most commonly prescribed anticancer drugs in a tertiary care teaching hospital. Indian J Pharm Pract. 2017; 10(4):270-5. [DOI:10.5530/ ijopp.10.4.55]

[11] Terwilliger T, Abdul-Hay M. Acute lymphoblastic leukemia: A comprehensive review and 2017 update. Blood Cancer J. 2017; 7(6):e577. [DOI:10.1038/bcj.2017.53] [PMID] [PMCID]

[12] McQuade R, Bornstein JC, Nurgali K. Anti-colorectal cancer chemotherapy-induced diarrhoea: Current treatments and side-effects. Int J Clin Med. 2014; 5:393-406. [DOI:10.4236/ijcm.2014.57054]

[13] Poddar S, Sultana R, Sultana R, Akbor MM, Azad MAK, Hasnat A. Pattern of adverse drug reactions due to cancer chemotherapy in tertiary care teaching hospital in Bangladesh. Dhaka Univ J Pharm Sci. 2009; 8(1):11-6. [DOI:10.3329/ dujps.v8i1.5330]

[14] National Cancer Institute (U.S.). Common Terminology Criteria for Adverse Events (CTCAE), Version 4.0. 2009. https://evs.nci.nih.gov/ftp1/CTCAE/CTCAE_4.03/CTCAE_4.03_2010-06-14_QuickReference_5x7.pdf.

[15] Sharma A, Kumari KM, Manohar HD, Bairy K, Thomas J. Pattern of adverse drug reactions due to cancer chemotherapy in a tertiary care hospital in South India. Perspect Clin Res. 2015; 6(2):109-15. [DOI:10.4103/2229-3485.154014] [PMID] [PMCID]

[16] Sahu RK, Yadav R, Prasad P, Roy A, Chandrakar S. Adverse drug reactions monitoring: prospects and impending challenges for pharmacovigilance. Springerplus. 2014 3:695. [DOI:10.1186/2193-1801-3-695] [PMID] [PMCID]

[17] Gibson RJ, Keefe DM. Cancer chemotherapy-induced diarrhoea and constipation: Mechanisms of damage and prevention strategies. Support Care Cancer. 2006; 14(9):890-900 [DOI:10.1007/s00520-006-0040-y] [PMID]

[18] Fracchiolla NS, Artuso S, Cortelezzi A, Pelizzari AM Tozzi P, Bonfichi M, et al. FarmaREL: An Italian pharmacovigilance project to monitor and evaluate adverse drug reactions in haematologic patients. Hematol Oncol. 2018; 36(1):299-306. [DOI:10.1002/hon.2451] [PMID]

[19] Behera SK, Kishtapati CR, Gunaseelan V, Dubashi B, Chandrasekaran A, Selvarajan S. Chemotherapy induced adverse drug reactions in cancer patients in a tertiary care 
hospital in south India. J Young Pharm. 2017; 9(4):593-7. [DOI:10.5530/jyp.2017.9.113]

[20] Kunwor P, Avinash C, Vishveshwara M, Madhavi Y, Vijayakumar $\mathrm{M}$. Anticancer drug induced infusion reactions: A single institute experience. Int J Pharm Sci Res. 2018; 9(9):3980-4. [DOI: 10.13040/IJPSR.0975-8232.9(9).3980-84] 